\title{
Equipoise and the Criteria for Reasonable Action
}

\author{
Emily L. Evans and Alex John London
}

I $\mathrm{n}$ her recent article, "Evidence, Belief, and Action: The Failure of Equipoise to Resolve the Ethical Tension in the Randomized Clinical Trial," Deborah Hellman gives a new twist to an old objection against clinical equipoise. ${ }^{1}$ Roughly, clinical equipoise is the requirement that there exist credible uncertainty in the expert medical community regarding the preferred treatment for a particular condition. This uncertainty is widely regarded as a necessary condition for enrolling participants in a clinical trial. The old objection is that clinical equipoise represents an overly permissive, and therefore morally unacceptable, mechanism for resolving the fundamental tension in clinical research between fidelity to the interests of the individual research participant, and fidelity to the statistical and scientific methods that are necessary to produce generalizable data in a reliable manner. ${ }^{2}$ Hellman's new twist on this objection utilizes some of the rudimentary architecture of Bayesian statistical theory to argue that clinical equipoise focuses our moral attention on the wrong issue. In particular, she claims that clinical equipoise permits an individual to be randomly assigned to a particular intervention when there is uncertainty within the larger medical community about the relative therapeutic merits of that intervention in comparison with the available alternatives.

Hellman's central thesis is that uncertainty of this type lies in the belief of clinicians about the relative therapeutic merits of a set of interventions in the $a b$ - stract, and that this does not address the central moral question: namely, whether allowing a patient to be randomized to these particular alternatives is consistent with a basic regard for the patient's health interests. Because uncertainty about the relative therapeutic merits of a set of interventions in the abstract might coexist with a determinate treatment preference for an individual with a specific medical profile, Hellman argues that the requirement that a trial begin in and be designed to disturb a state of equipoise is incapable of protecting the interests of individual trial participants. As a result, she holds that the equipoise requirement should be rejected as an inappropriate moral standard for regulating clinical research.

In the discussion that follows, we argue that Hellman's arguments do not take their own lessons far enough. Not only should equipoise be understood as a decision rule for determining when participation in a clinical trial is an admissible option for each $i n d i$ vidual trial participant, but equipoise should also be understood as playing an important role in establishing the proper relationship between clinical research and medical practice, and providing a normative standard for initiating and terminating clinical trials. The merits of adopting such a conception of equipoise are easily illustrated using the rudimentary statistical and methodological ideas that Hellman herself relies on in her critique. As a result, our response to Hellman is significant not only as a rebuttal of the most recent for-

Emily L. Evans, B.S., is a doctoral student in the department of Philosophy at Georgetown University. She received a bachelors of science degree in Ethics, History, and Public Policy with a minor in Health Care Policy and Management from Carnegie Mellon University in May 2005. Her work focuses on issues of justice in bioethics. Alex John London, Ph.D., is an Associate Professor of Philosophy at Carnegie Mellon University. He received his bachelors degree from Bard College and his PhD from the Department of Philosophy, The University of Virginia. His work focuses on foundational issues in research ethics, and he is co-author of Ethical Issues in Modern Medicine (McGraw Hill) 6th edition. In 2005 he was awarded a New Directions Fellowship from the Andrew W. Mellon Foundation. 
mulation of a longstanding objection to clinical equipoise, but also as a clarification of how the concept of equipoise should itself be understood. In particular, it is our contention that criticisms of the sort leveled by Hellman persist, in part, because there are a variety of different conceptions of equipoise within bioethics literature that are not often clearly distinguished. ${ }^{3}$ Responding to Hellman's critique provides an opportunity, therefore, to delineate a particular view of equipoise that has the special merit of being able to avoid an important set of objections.

\section{Hellman's Critique}

Hellman argues that there are three questions one can ask in response to any given data set, and clinical equipoise directs our attention to the wrong one. In order to evaluate this critique, it is important to have a clear grasp of the claims out of which her thesis is built. Consider, therefore, the following example that Hellman offers.

Suppose that a patient is not feeling well and visits his physician. The physician runs a series of diagnostic tests, and a test comes back with positive results for a particular medical condition (which we will call "M"). The first question the physician may have, therefore is: What does this evidence show? In this particular case, the evidence from the test indicates that the patient has $\mathrm{M}$. The physician's second question, however, is: Given this evidence, what should I believe? As Hellman correctly points out, the evidence from the test alone is insufficient for the physician to conclude that the patient has M. Rather, the physician must consider a number of other factors, including the sensitivity and reliability of the test, the base rate of the disease in the larger population, and risk factors that would help to establish whether the patient is at risk for having M. Because of these additional factors, there can be cases in which it is reasonable for the physician to believe that the patient does not have M, even in the face of a positive test. Hellman uses this possibility to show that, strictly speaking, what a particular set of data shows may not always correspond to what a clinician ought to believe. ${ }^{4}$

Hellman then argues that the most important question from the physician's standpoint is: Given this evidence, what should I do? Here again, Hellman correctly notes that what a clinician believes in such a situation may not be sufficient to determine her treatment recommendations. Instead, the latter question must take into account the various risks and benefits (the utilities) for the health interests of this patient that are as- sociated with the various courses of action. Even if the clinician believes it unlikely that her patient actually has $\mathrm{M}$, there exists a range of additional factors that might lead her to treat her patient for $\mathrm{M}$ nonetheless. For example, if $\mathrm{M}$ is a relatively serious condition, but the treatment is highly effective, inexpensive, and has a mild or benign side-effect profile, then it may be reasonable to treat the patient for $\mathrm{M}$ even if the clinician believes it to be unlikely that the patient has it. ${ }^{5}$

Hellman uses this example from the clinical context to underscore the idea that the answers to the second question, concerning what a clinician ought to believe in light of a particular set of data can diverge significantly from, and need not provide, the answer to the third question - what should the clinician do when faced with the particular benefits and burdens that a set of treatment options poses to the health of a patient?

Hellman maps this example from the clinical context onto the research context as follows. In the research context, the first question concerns what the existing
Because she understands clinical equipoise as focusing on what particular agents believe is the case, Hellman argues that this is a morally flawed standard for determining the permissibility of enrolling subjects into clinical research.

data reveal about the relative therapeutic merits of the relevant set of interventions. The second question concerns what physicians should believe about the relative therapeutic merits of these interventions in light of available data. Hellman claims that the state of uncertainty that constitutes clinical equipoise is formulated at this level. She holds that equipoise exists when experts in the clinical community are uncertain about what to believe concerning the relative therapeutic merits of the relevant set of interventions given the existing evidence about those interventions, the relevant beliefs about the reliability of that evidence, and the various properties of the condition in question.

Because she understands clinical equipoise as focusing on what particular agents believe is the case, Hellman argues that this is a morally flawed standard for determining the permissibility of enrolling subjects into clinical research. The relevant question concerning the health interests of the patient, she claims, is the third question - what should the clinician $d o$ for this particular patient in light of the various risks and benefits associated with each of the interventions in the relevant set. She therefore contends that the "equipoise 
justification for the randomized clinical trial ignores the distinction between the criteria for justified belief and the criteria for reasonable action." Even if a physician is uncertain about what to believe concerning the relative therapeutic merits of each treatment option, when confronted with a particular patient, careful consideration of the various costs and benefits associated with each treatment option will frequently generate a preference for one option over the other(s). As a result, Hellman claims that the equipoise requirement cannot resolve the tension between generating reliable data in a methodologically sound manner and protecting the interests of trial participants because it does not focus on the question most central to the participant's interests - given the available evidence and an individual's clinical profile, what is the best treatment for this particular individual?

Hellman's claim that equipoise focuses on the wrong question seems to be a reasonable inference in light of statements that are frequently made about equipoise in scholarly literature. For instance, Benjamin Freedman and others often claim that the equipoise requirement is synonymous with the requirement that a trial begin with an honest null hypothesis. ${ }^{7}$ This seems to support the idea that equipoise exists when there is honest disagreement in the clinical community about which treatment option under consideration is better for some condition in general. That is because whether an honest null hypothesis exists is something that is most commonly predicated of a trial in general, understood as a statement about what the existing evidence shows about the relative therapeutic merits of a set of interventions. It is less common to ask, for example, whether an honest null hypothesis exists for each individual trial participant.

Similarly, Hellman's understanding of the epistemic requirements of equipoise and her claim that these requirements will rarely be satisfied in practice is consistent with much of the literature on equipoise. Freedman, for example, in describing the view he calls "theoretical equipoise," states that equipoise exists when "the evidence on behalf of two alternative treatment regimens is exactly balanced." When describing Chalmers' position, Freedman also describes equipoise as the point at which the physician can say that "no evidence leans either way."9 Don Marquis and Richard Ashcroft also seem to focus on the importance of weighing evidence about medical interventions as such. Marquis writes that the arms of a study should be "therapeutically equivalent" and that it would be unethical for a physician to enroll a patient in a trial if he knew that the probability of Type I error (false-positive) was less than fifty percent.10 Ashcroft states the epistemic requirement of equipoise as follows: "Patients should be enrolled into clinical trials if there is no reliable epistemic reason (evidence) to favor one treatment over the other." ${ }^{11}$ Hellman concurs with this common understanding of the epistemic requirements and describes equipoise as obtaining when "the reasons to believe therapy $\mathrm{A}$ is better than $\mathrm{B}$ are evenly balanced by the reasons to believe that $\mathrm{B}$ is better than $\mathrm{A} .{ }^{\prime \prime}$

To be clear, therefore, Hellman's criticisms are based on a model of the equipoise requirement working as follows. First, members of the expert medical community must be uncertain about what to believe concerning the relative therapeutic merits of the interventions that are offered in various arms of clinical trials. If this uncertainty exists, then it is permissible to initiate a clinical trial and to enroll subjects who meet the general entrance criteria. This general view of equipoise is essential to Hellman's claim that the uncertainty requirement is not sufficiently responsive to the interests of the patient, and therefore cannot be used as a criterion for enrollment in a clinical trial. We want to underscore that as Hellman understands it, equipoise does not involve uncertainty concerning how to treat a patient in light of the various risks and benefits (utilities) associated with each relevant therapeutic option in relation to the patient's health interests.

\section{Equipoise as a Decision Rule}

Hellman's argument against clinical equipoise puts a new twist on older criticisms that essentially involve the conjunction of two claims. The first is that the clinical community is concerned with a policy judgment about which of a set of alternative treatment options is the best means of treating a given medical condition. The physician, however, is not setting policy. Her goal is simply to decide which treatment option is best for her specific patient. Whereas the clinical community is primarily concerned with what to believe about available treatment options, an individual physician is focused on acting in the patient's best interests in light of available treatment options. The second claim is that the epistemic standard required to make a decision about policy is much higher and more difficult to meet than the epistemic standard that is required to form a preference about the best treatment option for a particular patient. Thus, the goals and standards of the clinical community diverge from those of the individual physician in important ways.

Hellman's understanding of the equipoise requirement recognizes this distinction, and she makes a sharp separation between the criteria for belief (e.g., the setting of policy by the clinical community) and action (e.g., the clinical decision of the individual physician). Although we think her understanding of the equipoise requirement represents a reasonable inter- 
pretation of some of the literature, we think that the most interesting and important way of understanding this requirement avoids her objections. Moreover, we also think that a charitable reading of Freedman reveals that while he does not endorse this view explicitly, it is at least consistent with what he says, contrary to what Hellman argues.

According to the view that we endorse, the concept of clinical equipoise plays three important roles in clinical research. First, it establishes the proper relationship between clinical research and medical practice. Second, it provides a normative standard for determining when a clinical trial should be initiated and when it should be terminated. Third and finally, we argue that clinical equipoise also provides a decision rule for determining when it is permissible to enroll a particular patient in a clinical trial. Hellman fails to recognize the first of these roles and confuses the second and the third. This confusion prevents her from seeing the way that clinical equipoise can itself accommodate the very distinctions she uses to criticize it.

We begin, therefore, with the role that equipoise plays in what Hellman refers to as a criteria for reasonable action, particularly in the decision of how best to treat an individual patient. It is our contention that Hellman fails to see any role for equipoise in this regard because of a more general problem in the literature on equipoise that she simply recapitulates. This problem is a failure to properly distinguish and flesh out two closely connected, but nevertheless distinct, aspects to equipoise. The first aspect concerns whose uncertainty is relevant to determining whether or not equipoise exists..$^{13}$ In this regard, for example, Freedman argues that relevant uncertainty lies in the expert medical community, not in the mind of the individual clinician. Hellman's view may rest on the fairly natural assumption that the expert medical community can only be uncertain about the relative therapeutic merits of a set of interventions in the abstract. Perhaps more precisely, Hellman's claims against equipoise receive tacit support from an inability to see how the expert medical community could ever have an opinion about how to treat each individual trial participant.

This inability, in turn, stems from a failure regarding the second feature of equipoise, namely, how uncertainty should be understood. In particular, there is a general failure in the literature to distinguish two sources of uncertainty, what one of us has referred to elsewhere as clinical conflict, and agnosticism, the dominant model. ${ }^{14}$ Agnosticism occurs when "members of the expert medical community have not yet made determinate judgments about the relative therapeutic merits" of a set of interventions..$^{15}$ Perhaps the paradigm case of clinical agnosticism is when there is not yet enough data about a novel therapy to convince the medical community about its relative merits in comparison with other, more established, treatment options. The expert medical community is agnostic in the sense that no consensus opinion has been formed or enunciated. That judgment awaits better data, and the goal of research is to provide the data that will resolve this question.

Hellman, like other critics before her, is correct in her assertion that even when uncertainty of this sort exists in the expert medical community, it may be perfectly rational for the individual clinician to have a decided treatment preference when faced with a particular patient. In fact, the practical nature of the clinician's job actually requires the clinician to make the best decision possible for the particular patient and this practical imperative militates against remaining agnostic about the relative therapeutic merits of the available treatment options.

Unfortunately, however, clinical agnosticism is not the only source of uncertainty in medicine. Uncertainty may also exist precisely because different members of the expert clinical community have definite but conflicting preferences about the treatment alternatives available for treating a patient. Consider, for example, a patient, $\mathrm{P}$, who has medical condition, $\mathrm{C}$, and visits his physician for treatment. $\mathrm{P}$ learns that there are two competing treatments for C, let's call them A and B. P's physician explains the various benefits and burdens associated with each intervention and then explains that when all things are considered, he, the physician, would recommend A. Because choosing a treatment is an important decision, $\mathrm{P}$ then seeks a second opinion from a physician who, after a thorough examination, explains that, all things considered, she prefers treatment B over A. Now P finds himself with conflicting medical advice. Let us assume further that $\mathrm{P}$ considers the medical credentials of each physician and finds that both are experienced, well-credentialed medical experts and, moreover, the views of each are mirrored by a larger disagreement about how to treat $\mathrm{C}$ in the broader medical community.

This is not a case of agnosticism since different segments of the expert medical community have not withheld their "all things considered" judgment. Rather, they have come to conflicting judgments about the merits of treatments A and B, not in the abstract, but as treatments for P. Similarly, this is not a case in which the treating physicians or $\mathrm{P}$ are indifferent between $\mathrm{A}$ and B. Rather, each physician has a definite preference for one treatment option. However, those decided preferences are in conflict. ${ }^{16}$ If the conflict is extreme, each physician may even council $\mathrm{P}$ against undertaking the mode of treatment preferred by the other physician. 
Clinical conflict provides the clearest mechanism by which Freedman's conception of clinical equipoise, with its epistemic standard of consensus in the expert medical community, can be seen as a decision rule for determining whether a particular patient may permissibly be entered into a specific clinical trial. In particular, as in the case of $\mathrm{P}$ above, if at least a reasonable minority of expert clinicians would recommend A over $\mathrm{B}$ as treatment for $\mathrm{C}$, while others recommend $\mathrm{B}$ over $\mathrm{A}$, then we hold the option of random assignment of $\mathrm{P}$ to a trial of either A or B admissible.

In fact, one of us has previously argued that it would be unjustified paternalism to prevent $P$ from enrolling in a trial of A or B under these circumstances..$^{17}$ Such a restriction would amount to arbitrarily limiting P's treatment options on the basis of one set of clinical opinions when there exists at least a reasonable minority of expert clinicians who offer conflicting recommendations. If a reasonable minority of expert clinicians would recommend $\mathrm{A}$, and a reasonable minority of expert clinicians would recommend B, then both are admissible treatments. In such a case, the "mixed" option of being randomly assigned to either A or B is also admissible, and $\mathrm{P}$ should therefore be offered the option to participate in the clinical trial.

In our view, there are two conditions under which it is permissible to offer a patient participation in a clinical trial: if the expert medical community is agnostic about the relative therapeutic merits of the available interventions for that patient, and if individual physicians have determinate treatment preferences for that individual, but those preferences are in conflict with one another. Notice, therefore, that this standard can accommodate exactly the situation that Hellman envisions. That is, it might be permissible to conduct a clinical trial because either a state of agnosticism or state of conflict exists in the larger medical community about the relative merits of a set of interventions. But it might not be permissible to enroll a particular patient in that trial if, in fact, there is no agnosticism or conflict about how to treat that condition in this particular person. If, for example, the clinical community were divided over the relative merits of $A$ and $B$ for treating $C$, but $\mathrm{P}$ had a complicating medical condition that would be exacerbated by the side-effects of A, then there may be no disagreement that $P$ should receive treatment $B$. In this instance, it would not be permissible to offer participation to $\mathrm{P}$ precisely because equipoise would not obtain in his case.

Moreover, the state of clinical conflict helps to clarify the role of clinical equipoise in determining when it is permissible to terminate a clinical trial. As Hellman recognizes, when faced with a patient, the clinician may have a determinate preference for one treatment option over the others and thus may not view participation in a clinical trial as a permissible option. On this model, it would only be permissible to offer enrollment to the patient if the clinician was unable to determine whether one option weakly dominates the others. This might happen, for example, if the clinician was uncertain about the probabilities and utilities that attach to the outcomes associated with each of the available interventions. However, even if this state of uncertainty would permit a trial to begin, once data began to emerge from the trial, such a state of uncertainty would be disturbed, and it would no longer be permissible to continue the trial. Even if it permits trials to begin, this view of clinical equipoise is so fragile as to prevent trials from producing statistically significant data.

However, the fragility of this view stems precisely from a failure to recognize the role of clinical conflict in determining equipoise. Assume that the interim data from the trial are sufficient to lead at least a reasonable minority of clinicians to prefer treatment $\mathrm{A}$ over $\mathrm{B}$ for patient $\mathrm{P}$. If, after assessing this data, at least a reasonable minority of clinicians would continue to recommend $\mathrm{B}$ over $\mathrm{A}$ for $\mathrm{P}$, then it is permissible for $\mathrm{P}$ to continue in the trial because there is still a state of conflict about treatment in the expert medical community. If, on the other hand, the data are sufficient to bring about a reasonable consensus among expert clinicians about the superiority of either A or B as a treatment for $\mathrm{P}$, then it would no longer be permissible for $\mathrm{P}$ to participate in the trial because equipoise would have been disturbed. ${ }^{18}$

On our view, therefore, whether it is permissible to initiate and to continue a clinical trial is a function of whether there exists a state of agnosticism or clinical conflict about how to treat a sufficient number of individual patients to warrant the initiation or continuation of a trial. Obviously a thorough defense of our view would require a more detailed explication and defense of some of these claims. For our present purposes it is sufficient, however, simply to illustrate that clinical equipoise can be understood in a way that contradicts Hellman's central thesis and that such a position has sufficient merit as to be worth pursuing in more detail.

Moreover, it is also worth emphasizing that too little attention has been paid to Freedman's recognition that equipoise may exist for the individual patient when physicians have determinate but conflicting treatment preferences, because it is "largely a matter of chance that the patient is being seen by a clinician with a preference for B over A, rather than by an equally competent clinician with the opposite preference." ${ }^{\prime 19}$ It is our claim, contrary to what Hellman has argued, that in this situation equipoise obtains relative to the in- 
dividual patient as long as a "reasonable minority of informed and reflective expert clinicians would offer advice to patients that conflicts with the advice of the majority."20

We agree with Hellman, therefore, that wise clinical decisions - particularly the recommendation of a specific course of treatment for an individual patient, including the option of enrolling in a clinical trial - require the integration of three elements. First is the available data about the interventions in question. Second, one must assess this data in light of one's beliefs about the medical condition, the relative therapeutic merits of a set of interventions, the prevalence or base rate of the disease, and the sensitivity and specificity of the tests. Third, the decision about how to treat a given patient must bring this information to bear on the particular situation of the individual patient, including his specific clinical profile and the relative burdens and benefits of the various treatment options for his case. Unlike Hellman's view, however, our view of equipoise plays an important role in integrating these three elements of wise decision-making at multiple levels in the clinical community. In other words, our conception of equipoise can be used as a decision rule for individual clinicians attempting to make wise treatment decisions, as a guide to the expert medical community for setting responsible evidence-based standards for medical practice, and as a model that the research community can use in determining how to advance the state of knowledge so as to improve the future standard of care.

The following diagrams illustrate the role that equipoise plays in integrating these components of wise decisions (Figures $1-3$ ). In each figure, each of the three

\section{Equipoise as a Decision Rule: Research,Treatment, and Theory}

\section{Three Components of a Wise Decision}

- Available Data about the relative therapeutic merits of a set of interventions

- Background Beliefs about the particular medical condition and the relative therapeutic merits of a set of interventions

- Factors Influencing Clinical Decisions include the clinical profile of the particular individual and the individual's own values

Figure 2:

\section{The Wise Treatment Decision}

$$
\text { Background Beliefs }
$$

Available Data

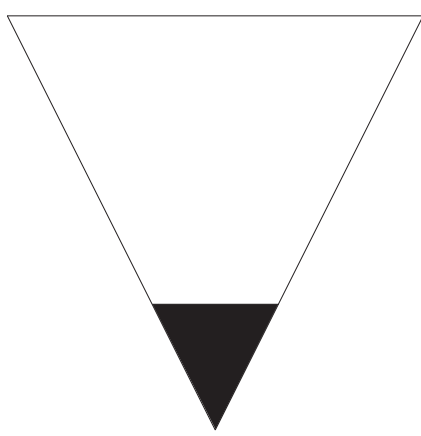

Factors Influencing Clinical Decisions

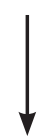

Treatment Decision \&

Equipoise Requirement
Figure |:

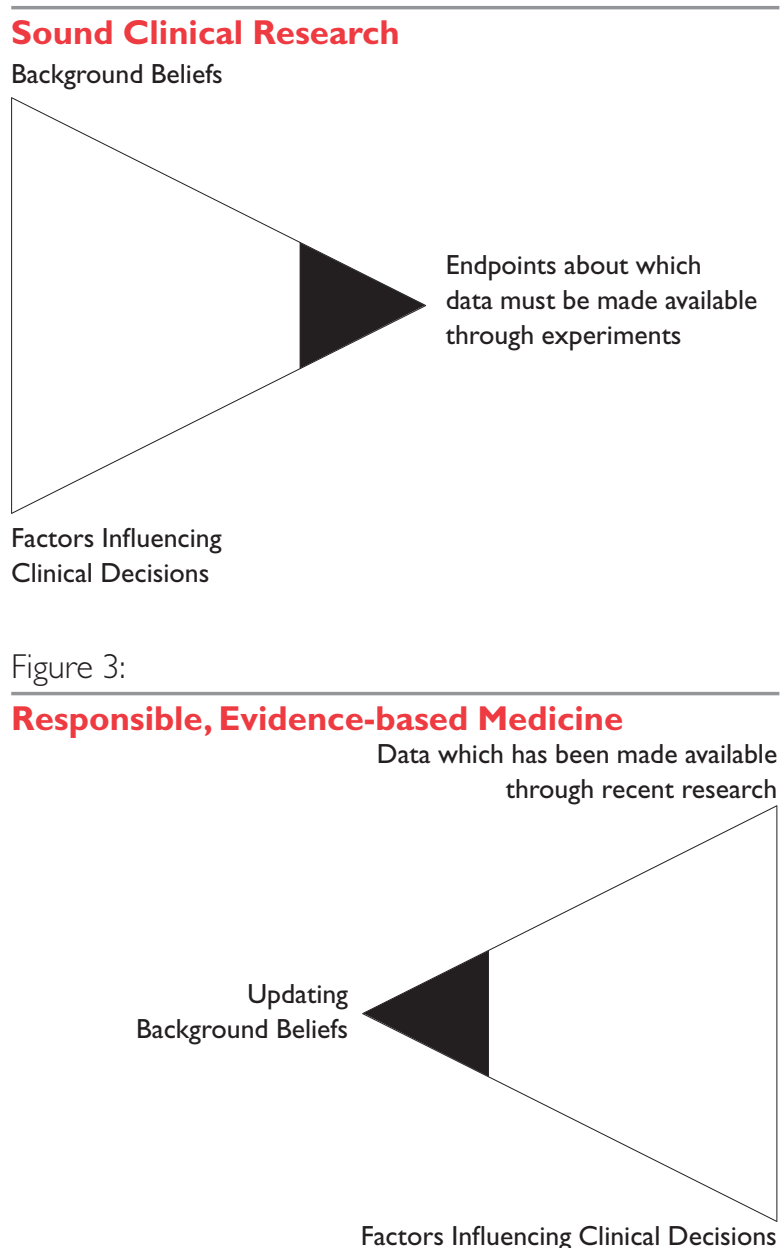


corners of the isosceles triangle represents a key aspect of sound decision making - available data, background beliefs, and the factors influencing a particular clinical decision. One tip of the triangle is then darkened to indicate the main aspect of the question at hand and the influence of the other components on it. By ratcheting the triangle, we can illustrate the way in which these three elements must be integrated, shifting the focus to a different aspect of a decision and showing the influence of the other aspects on it.

Figure 1 represents the factors that go into sound decision making about when to undertake or terminate a particular research initiative, and how equipoise plays a role in these decisions. The orientation of the triangle indicates that the two elements at the base play a fundamental role in determining which specific questions need to be studied in a clinical trial. If, for example, there is a state of conflict in the expert medical community about how to treat specific patients with the medical condition in question, then the trial should be designed in such a way that it will resolve this conflict. In other words, in order to design a trial that will advance the state of medical practice, one must know what factors influence the treatment decisions of clinicians and what background beliefs are relevant to those decisions so that the trial can study the issues that actually influence the clinical practice of physicians. This figure, therefore, illustrates the role of equipoise in determining what kind of data will be most relevant to medical practice, and have the greatest social value.

Freedman is explicit in articulating this role for clinical equipoise. In fact, he defends clinical equipoise as a standard that gives priority to "pragmatic" clinical trials, meaning that trials should be designed, run, and interpreted "in such a way as to maximize clinical relevance and the goals of medical practice rather than methodological rigor and the goals of theoretical science."21 But Hellman's analysis of his position is so uncharitable that it is effectively unable to explain why Freedman thought that equipoise would actually be able to achieve this objective. Her interpretation of equipoise allows clinical trials to be initiated and designed to generate data about an issue that may not be essentially connected to the decision making process of actual clinicians.

Thus, the diagram in figure 1 is intended to illustrate simply that it is precisely the existence of conflict or uncertainty about how best to advance the health care interests of particular patients that not only creates the occasion on which to conduct a clinical trial, but also helps to determine which particular questions are addressed by that trial. Our point is that a proper understanding of the role of clinical equipoise cannot separate these elements in the way that Hellman's analysis presupposes.

This point is amplified by figure 2, which illustrates the process of sound clinical decision making about whether or not participation in a clinical trial is an admissible option for a particular patient. Here, the elements at the base of the triangle are brought to bear on the case of the particular individual in light of his or her specific clinical characteristics. If, when these factors are evaluated in light of the available data and relevant beliefs, the expert medical community believes that one treatment option is best for this patient, then

\section{Hopefully, clarifying these points will serve to advance the state of the debate about equipoise by focusing attention on versions of this requirement that are the most sophisticated, operationally precise, and morally defensible.}

participation in the trial is not an admissible option. If, however, the expert medical community is conflicted about which of the available interventions is best for this particular patient, then the patient may be offered participation in the clinical trial as an admissible option.

To be clear, even though the expert medical community may be conflicted about how to treat a particular patient, that patient may not be similarly conflicted when faced with a choice of treatments. In particular, if she has firm values that make one treatment option appear particularly unattractive, she may not want to risk any chance of being randomly assigned to that option. It is important to recognize, therefore, that clinical equipoise represents a standard for determining which trials it is permissible to offer to individual patients. Whether those patients are willing to participate will depend on their own values. ${ }^{22}$

Finally, figure 3 illustrates the factors influencing responsible, evidence-based medicine. If a clinical trial is designed and executed in such a way as to disturb or eliminate a state of equipoise, then it will have generated sufficient evidence to resolve either clinical agnosticism or clinical conflict at its termination. When this occurs, clinicians must revise or update their background beliefs if necessary, and they must make use of this newly available data in the treatment of future patients. According to Paul Miller and Charles Weijer, physicians have "a moral obligation to keep up to date with scientific advances in medicine, and when sub- 
stantial evidence is presented, to change their practices accordingly." ${ }^{23}$ In updating their beliefs, physicians establish a new relevant set of background beliefs and factors influencing clinical decisions that will be important both to future treatment decisions and to future decisions about which questions should be put on the research agenda.

In contrast to Hellman's claim that equipoise cannot serve as a normative guideline for determining when participation within a clinical trial is an admissible option for each individual trial participant, we have argued that it should be understood as a decision rule for that very purpose. On our view, because the concept of equipoise plays a fundamental role in integrating the three key aspects of sound clinical decision making, it provides a normative standard for initiating and terminating clinical trials, and establishes the proper relationship between clinical research and medical practice. It therefore has an integral role to play in the decisional process of sound clinical research, wise treatment decisions, and responsible, evidence-based medicine. We have also tried to indicate that there is strong evidence that Freedman recognized the central elements of this account. He was not as precise as he might have been about important points, such as the role of conflict in determinations of equipoise, and while he did not endorse this account, what he does say at least appears to be consistent with it.

\section{Other Views that Avoid Hellman's Objection}

It is worth noting, in conclusion, that there are at least two other contemporary views that recognize the role of equipoise in determining whether it is permissible for individual participants to enroll in a clinical trial and which therefore avoid Hellman's objections to the use of equipoise in this capacity. J. A. Chard and R. J. Lilford's theory of equiphase makes use of a very traditional application of utility theory to determine whether enrollment in a clinical trial is an admissible option for a particular patient. ${ }^{24}$ Alternatively, although the Kadane-Sedransk-Seidenfeld (KSS) model does not explicitly use the language of equipoise, it makes explicit use of conflict in the recommendations of expert clinicians as a criterion for enrolling individual participants into a clinical trial. ${ }^{25}$ To understand how these views avoid Hellman's objections, we will briefly sketch the underlying assumptions and implications of each approach.

According to Chard and Lilford, equipoise plays two important roles in clinical decision making. First, it provides the standard for initiating clinical trials. Chard and Lilford argue that "collective equipoise," or equipoise at the level of the expert medical community, must obtain before any clinical trial can be approved.
Once collective equipoise obtains and a trial begins, it is "individual equipoise," or equipoise in the mind of the individual clinician, that makes it ethically permissible to offer a particular patient enrollment in the trial. Individual equipoise thus becomes the rule for determining when it is permissible to offer a particular individual enrollment in a clinical trial. To determine whether individual equipoise exists, the values of the patient are mapped onto a utility scale containing the clinician's suggested probabilities for the different outcomes contingent upon each intervention option. The expected utilities of each intervention can then be calculated, with equipoise obtaining when competing interventions have equal expected utilities. Because a patient and a clinician may have a range of utilities and probabilities, respectively, equipoise may obtain along a range of expected utilities; Chard and Lilford refer to this range of outcomes along which the patient and clinician are indifferent as "equiphase" (as opposed to equipoise, which suggests a single point of indifference). By allowing equipoise to obtain along a range of expected utilities, Chard and Lilford further underscore the importance of focusing on the clinical profile of the individual and his values and preferences when selecting admissible interventions (including enrollment in a clinical trial).

Because Chard and Lilford specifically formulate individual equipoise as a rule for determining whether it is permissible to offer an individual enrollment in a clinical trial, they avoid Hellman's objection that equipoise cannot be applied at the level of the individual patient. However, we think that their view suffers from several significant drawbacks, only one of which we will mention here. In particular, Chard and Lilford state that collective equipoise may obtain when "individual [physicians] have a preference for one treatment... but are balanced by others with the opposite view, so overall the profession is equipoised," and they claim that when such disagreements exist it is permissible to initiate a clinical trial. ${ }^{26}$ However, when it comes to the permissibility of enrolling a particular patient into a clinical trial, they formulate equiphase only in terms of the single utility function that is constructed out of the individual physician's probabilities and the patient's utilities. Their theory is, therefore, open to the objection from paternalism that we mentioned above. Namely, it does not recognize participation in a clinical trial as an admissible option for a particular patient when there is a conflict in the treatment recommendations that different expert clinicians would make to the individual patient. ${ }^{27}$

The KSS model, on the other hand, is very explicit about modeling the judgments of several experts whose views are represented views that are held within the 
expert community when deciding which treatment to assign to individual patients. Although the model does not use the language of equipoise, it is predicated on a view of clinical trials as a mechanism for resolving conflict or uncertainty within the expert medical community while ensuring that the interests of the individual patient are not compromised. Since this goal reflects the basic moral premise underlying the equipoise requirement, we believe their model should be understood as a way of making this concept operational.

Operationalizing equipoise as a decision rule, the KSS model (1) defines a set of admissible therapies for any given patient, ensuring that the patient receives a treatment that is determined to be the best for them by at least one physician expert and (2) continually updates the opinions of experts as the trial progresses in light of the data being generated. Kadane describes the model as follows:

The proposal then is that unless at least one expert (as modeled on the computer) finds a treatment to be the best for someone with the predictor variables of the patient, it will not be assigned. Thus, if only one expert finds a treatment the best for a patient of a given type, it could, under this rule, be assigned to that patient. Within this constraint of admissible treatments, patients may be assigned to treatments in any one of many ways - randomly, or to maximize balance on important predictor variables, or in any other way. ${ }^{28}$

This passage reveals many important aspects of the KSS trial design. For our present purposes it is sufficient to note that, by modeling the conflicting treatment recommendations of several clinicians, this model effectively uses equipoise at the level of the clinical community to determine the set of admissible interventions for a particular individual. Because the patient will be assigned to an intervention that has been identified as admissible for him, enrollment in the trial ensures that the individual receives an intervention that would be recommended for her by at least a reasonable minority of expert clinicians.

Although there remain limitations to the KSS model, ${ }^{29}$ it is important to note for our purpose simply that Hellman's objections to the use of equipoise for determining whether it is permissible to offer an individual enrollment in the clinical trial do not apply to the KSS model. Although we disagree with Chard and Lilford, their view is similarly immune to Hellman's charge. Both of these views, however, have the singular merit of using precise decision-theoretic models to give explicit, action-guiding content to the concept of equipoise. Hopefully, clarifying these points will serve to advance the state of the debate about equipoise by focusing attention on versions of this requirement that are the most sophisticated, operationally precise, and morally defensible.

References

1. D. Hellman, "Evidence, Belief, and Action: The Failure of Equipoise to Resolve the Ethical Tension in the Randomized Clinical Trial," Journal of Law, Medicine छ Ethics 30 (2002): 375-380.

2. See e.g., S. Hellman and D. S. Hellman, "Of Mice but not Men: Problems of the Randomized Clinical Trial," N. Engl. J. Med. 324 (1991): 1585-1591; D. Marquis, "Leaving Therapy to Chance," The Hastings Center Report 13, no. 4 (1983): 40-47; and F. Gifford, "The Conflict between Randomized Clinical Trials and the Therapeutic Obligation," The Journal of Medicine and Philosophy 11 (1986): 347-366.

3. A. J. London, "Clinical Equipoise: Foundational Requirement or Fundamental Error?" Forthcoming in B. Steinbock, ed., The Oxford Handbook of Bioethics (New York: Oxford University Press, in press). See also A. J. London, "Two Dogmas of Research Ethics and the Integrative Approach to Human-Subjects Research," Journal of Medicine and Philosophy, forthcoming.

4. See Hellman, supra note 1.

5.Id.

6. Id., at 379 .

7. B. Freedman, "Equipoise and the Ethics of Clinical Research," $N$. Engl. J. Med. 317 (1987): 141-145. See also P. B. Miller and C. Weijer, "Rehabilitating Equipoise," Kennedy Institute of Ethics Journal 13, no. 2 (2003): 93-118.

8. Id., at 143 .

9. Id.

10. See Marquis, supra note 2, at 40.

11. R. Ashcroft, "Equipoise, Knowledge, and Ethics," Bioethics 13 (1999): 314-326, at 317.

12. See Hellman, supra note 1, at 375.

13. See London, supra note 3.

14. Id.

15. Id.

16. It is worth emphasizing that indifference is a distinct relationship from both agnosticism and conflict. As we understand agnosticism, this is a state in which an agent has not yet endorsed an allthings-considered assessment of the relative merits of options for choice. This state provides the occasion for further inquiry and analysis. Indifference, on the other hand, represents a judgment that the options available to the agent are equivalent in value for the purposes of the choice problem at hand. If one is indifferent between two cans of cola, one is not in a state of agnosticism about which can would be most preferred after further inquiry. Rather, there is no need for further inquiry because one has judged that one can is as good as the other for the purpose at hand. Conflict, however, arises when an agent recognizes more than one value or commitment as relevant to determining what ought to be done, each value or commitment provides a determinate ranking or evaluation of the options, but these evaluations cannot be jointly satisfied. See I. Levi, Hard Choices (New York: Cambridge University Press, 1986). In the technical parlance of decision theory, when a set of values is conflicted, the set as a whole lacks the property of completeness which can be paraphrased as the property that for any pair of objects $\mathrm{x}$ and $\mathrm{y}$ in an agent's choice set that are not identical, either (a) $\mathrm{x}$ is at least as good as $\mathrm{y}$ or (b) y is at least as good as x. Conflict should not, therefore, be equated with indifference since in conflict, neither (a) nor (b) is being asserted, whereas in the case of indifference, both are. See also A. Sen, Collective Choice and Social Welfare (Amsterdam: Elsevier Science Publishers, 1970). As Isaac Levi has argued, one response to conflict might be to assume a position of agnosticism and attempt to resolve the conflict through further inquiry. Alternatively, if conflict is intransigent, or if time is limited, one might have to engage in decision making under unresolved conflict. It is our contention that clinical equipoise should be understood 
as a method for making decisions under unresolved conflict. We therefore think it is a mistake to equate the state of equipoise with indifference or to limit the use of randomization to cases in which either the broader medical community, the treating physician, or the individual research participant is indifferent between the available treatment options. For an example involving the latter case, see R. Veatch, "Indifference of Subjects: An Alternative to Equipoise in Randomized Clinical Trials," in Bioethics, E. Frankel Paul, F. D. Miller, Jr., and J. Paul, eds., (Cambridge: Cambridge University Press, 2002): 295-323.

17. See London, supra note 3.

18. Id.

19. See Freedman, supra note 8 , at 144 .

20. See London, supra note 3. Here our argument follows Freedman, supra note 7; J. B. Kadane, ed., Bayesian Methods and Ethics in a Clinical Trial Design (New York: John Wiley \& Sons, Inc., 1996); and Miller and Weijer, supra note 7.

21. F. Gifford, "Freedman's 'Clinical Equipoise' and 'Sliding-Scale AllDimensions-Considered Equipoise," Journal of Medicine and Philosophy 25 (2000): 399-426, at 404.

22. Similar efforts to accommodate patient preferences are offered by R. Veatch, supra note 16; and by Miller and Weijer, supra note 7 .
23. Id., at 108.

24. J. A. Chard and R. J. Lilford, "The Use of Equipoise in Clinical Trials," Social Science and Medicine 47, no. 7 (1998): 891-898.

25. See Kadane, supra note 20.

26. See Chard and Lilford, supra note 24, at 891-892.

27 . Miller and Weijer, supra note 7, offer a more conceptual rendering of Chard and Lilford's decision-theoretic theory, requiring that (1) equipoise in the expert medical community obtain before a trial is initiated and (2) equipoise in the mind of the individual clinician obtain before a patient can be offered enrollment in that trial. Similarly, Miller and Weijer argue that the "particular circumstances of the particular patient" must be the focus of the physician's treatment decision. Moreover, they acknowledge that data alone is insufficient to make treatment recommendations, but argue that the individual physician's evidence, beliefs, and values invoked in the decision must be clinically significant and open to "professional, impersonal validation."

28. See Kadane, supra note 20, at 9.

29. See Kadane, supra note 20, particularly chapter 2. 